\title{
A fast word search algorithm for the representation of sequence similarity in genomic DNA
}

\author{
Christophe Lefèvre and Joh-E.Ikeda \\ Genosphere Project, ERATO, JRDC, Tokai University School of Medicine, Isehara, \\ Kanagawa 259-11, Japan
}

Received September 21, 1993; Revised and Accepted December 22, 1993

\begin{abstract}
Representation of sequence similarity by dot matrix plots is a method widely used for comparing biological sequences. The user is presented with an overall view of similarity between two sequences. Computation of this plot has been reconsidered here. An improvement is proposed through the preprocessing of the data into an automaton recognizing the word structure of a sequence. The main advantage of this approach is to systematically eliminate the repetitions during word comparison. Simple heuristics are also considered to greatly speed up pattern matching. As a result, large sequences are handled very efficiently. This is illustrated by a comparison of large genomic DNA. The algorithm has been implemented in an interactive application on a microcomputer.
\end{abstract}

\section{INTRODUCTION}

The visual representation of sequence relatedness using dot matrix similarity plots is one direct way to compare biological sequences $(1-8)$. The principle is simple. For two sequences of length $N$ and $M$, a dot matrix of $N$ rows and $M$ columns is computed. A 'dot' indicates a match between the letter, word or pattern at the positions corresponding to the row and column in the respective sequences. Extended regions of similarity tend to form diagonals, and the pattern recognition abilities of the human brain allows the user to identify those regions instantly. This technique is rather subjective and only semi-quantitative but presents an overall picture of sequence similarity. As the biological interests in sequence similarity involve many different molecular aspects, the biologist sometimes favors this suggestive approach over more rigorous similarity searches that may overlook some important local features. This aspect is particularly relevant to the consideration of larger sequences such as genomic DNA where local similarity can be found at different levels.

As DNA sequencing enters the era of genome sequencing, improved algorithms are needed for the manipulation and analysis of this new, potentially monumental, data. Here, in an attempt to achieve efficiency on the microcomputer, the problem of the computation of dot matrix similarity plots in 'minimal' time is reconsidered. Most dot matrix algorithms execute with a time proportional to the product of the lengths of the sequences being compared (1-8). The technique can be improved through the use of oligomer tables containing indexes to the positions of occurrence of a list of oligomers (9). Here, this principle is developed further through the compilation of an original data structure; the position end-set tree (10), which can be viewed as an automaton recognizing the word structure of a sequence and can be used as a flexible and general oligomer table. As this data structure requires a substantial amount of memory, the progress in the manufacture of memory device has made this approach realistic, even at the scale of the microcomputer. Our tree search algorithm uses this data structure to localize word matches between two sequences directly comparing words, rather than sequence positions. Although it is generally believed that word search approaches to sequence comparison are impractical due to the abundance of words that can be formed from a sequence, it is shown here that this is not the case. On the contrary, the reconsideration of the concept of similarity in biological sequences leads to the implementation of simple heuristics resulting in better control of computational complexity. This technique performs satisfactorily in the detection of similarity in DNA sequences. Large sequences can be handled more efficiently as the time penalty may grow slower than the product of the sequence lengths. This is illustrated with two examples: a human genomic fragment encoding the growth hormone gene cluster and the complete yeast chromosome III sequence.

\section{METHOD AND APPLICATION}

All computations were done on a Macintosh II-fx equipped with 132 Megabytes of random access memory. The Program is written in object oriented $C$ language supported by THINK $C^{\text {TM }}$ for Symantec ${ }^{\mathrm{TM}}$ corporation (version 5.0).

The position tree: an index table to word occurrence A position tree is represented in Figure 1. Words are represented by a node. The sequence of edge labels on the path from the root to a node spells the word represented by this node. The set of leaves represents the set of shortest non repeated prefix of all unique words in the sequences (also called the position identifiers of the sequence). The leaves are labeled with the position of the word that they represent. Every repeat in the sequence is represented by a single internal node. A space economical variation of this position tree was recently described (10). It is more appropriate for the compilation of biological sequences. Space requirement is linearly proportional to the sequence length and an algorithm for a compilation in linear time 
has been presented (10). This index to the sequence provides rapid string searching for the extensive localization of words (11) or patterns in a main text (12). The positions of occurrence of a word can be obtained from the labels of the set of leaves of the sub-tree rooted at the corresponding node. This can be done with a simple recursive procedure requiring a number of steps proportional to the word occurrence, independently of the length of the sequence (13). This fast access to the positions of a word occurrence led us to consider the use of the position tree for the representation of sequence similarity by dot plots, where dots are placed in the matrix at the intersections of the positions of word matches in two different sequences.

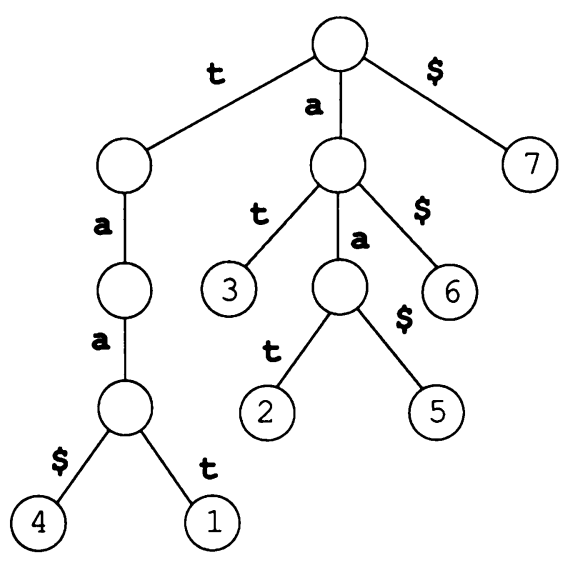

Figure 1. Position tree for the sequence 'taataa\$'.

\section{Basic algorithm: the perfect match problem}

A position tree is precompiled for each of the sequences. The user sets a tuple parameter $T$ defining the length of words to be taken as matches. With a tree transversal algorithm, the edge labels of the first position tree are compared with those from the other sequence. Suppose that a sub word $w$ of length $k$ in sequence $\mathrm{S} 1$ represented by node $\mathrm{N} 1$ matches sub-word $w$ in sequence $\mathrm{S} 2$ represented by node N2. As illustrated in Figure 2 several cases may occur. (1) If $k=T$ then dots should be placed in the matrix at all the intersections of the occurrence of $w$ in sequence S1 and S2. These positions can be obtained from the labels of the set of leaves of the sub-trees rooted at N1 and N2. (2) If $k<$ $T$, three cases can be considered. (i) If $\mathrm{N} 1$ is a terminal node, then $w$ has a single occurrence in sequence $\mathrm{S} 1$ at the position of the label of N1. The $T-k$ remaining symbols from the end of $w$ in sequence $\mathrm{S} 1$ can be match against the sub-tree rooted at $\mathbf{N} 2$. If a leaf is encountered in the sub-tree before a match length $T$, it identifies a single occurrence in sequence $\mathrm{S} 2$ and a direct reference to the symbols in the sequence can be made to check if a match of $T$ letters can be achieved. This is equivalent to the classic problem of the localization of a word in a sequence (11).(ii) If $\mathrm{N} 2$ is a terminal node, this is the same situation as described in (i) where N1 and N2 are interchanged. (iii) When $\mathrm{N} 1$ and $\mathrm{N} 2$ are internal nodes in the position tree, they represent repeats in S1 and S2. The edge labels extruding from N1 and $\mathrm{N} 2$ are compared systematically and, when a match is found, the edges lead to the node ND1 and ND2 with which the all process can be reiterated. Finally, because words of increasing length are being considered and, the case where $k=T$ terminates a path in the position tree, $k$ does not grow greater than $T$ during this recursion.
1

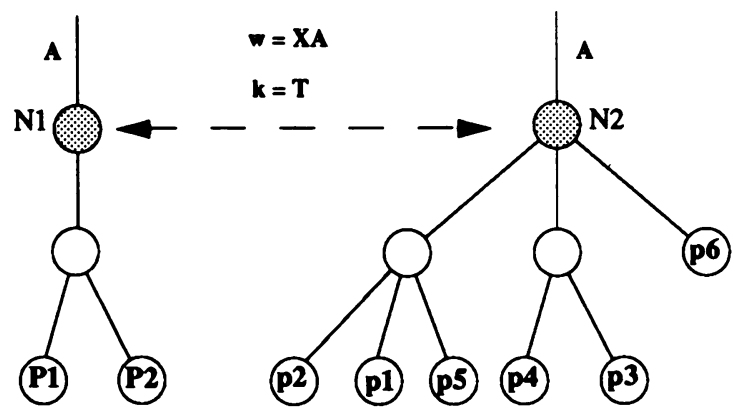

positions of occurence of $\mathbf{w}$

in $\mathrm{S} 1$

$\{\mathbf{P 1}, \mathbf{P 2}\} \quad \mathrm{X} \quad\{\mathrm{p} 2, \mathrm{p} 1, \mathrm{p} 5, \mathrm{p} 4, \mathrm{p} 3, \mathrm{p} 6\}$

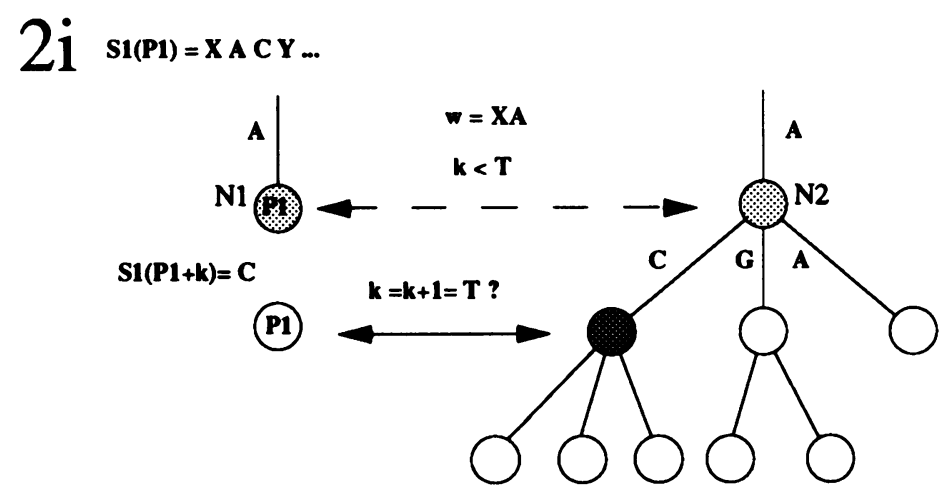

2iii

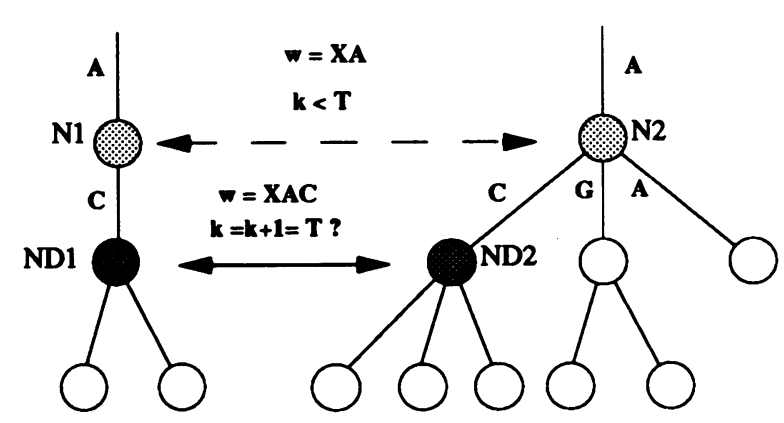

Figure 2. Word search algorithm. Illustrations of different cases encountered during the perfect matching of two position trees (see text for details). 
This algorithm is easily implemented by recursive programming. For example, the procedure Match2trees is given below and will provide all the coordinates of the points in a dot matrix when called with the roots of the position trees (representing the empty word) as in the call: 'Match2trees(root1, $\operatorname{root} 2,0)$ '. Match2trees (Node1, Node2,k)
.if $k=T$ then

.collect positions of occurrence of word $w$ from the sub-trees rooted at Node1 and Node2. .return

if Nodel is a leaf then .match the $T-k$ symbols following the word represented by Node1 with sub-tree rooted at Node2 .return

if Node2 is a leaf then . match the $T-k$ symbols following the word represented by Node 2 with sub-tree rooted at Nodel.

for all edges leaving Node 1

let ND1 be the node this edge leads to

if there is an edge leaving Node2 with the same label

then let ND2 be this node this edge leads to .Match2trees (ND1, ND2, $k+1)$

.return

If we ignore the time taken by the tree transversal, this algorithm executes in time proportional to the number of matches or dots in the dot plot. This appears as an interesting behavior, as the display for significant similarity in biological sequences ultimately consists of a search for a few 'best' matches.

\section{Optimizing graphic operations}

If there are few matches, they are trapped in little more time that is necessary to transverse the position tree. When there are many word matches (several matches per word as depicted in Figure 2, case 1), it is economical to remember a line (a graphic stamp) during the transversal path over the sub-tree of the horizontal sequence. In the graphic memory device, the line can be stamped directly at all the positions covered by the path of the vertical sequence. The effort of drawing all points one by one is saved.

\section{Application: strong similarity in a human gene cluster}

In Figure 3, two dot plots were obtained with a genomic fragment encoding the human growth hormone gene cluster. It is a large genomic sequence with a lot of internal similarity. This cluster contains 5 related genes which evolved recently through a complex series of duplication and gene conversion events. The genes are contained in a 66 kilobases (kb) DNA fragment and arranged in the same orientation (14). A dot represents a perfect match of 15 nucleotides. In Figure 3a, the sequence is compared to itself. The main diagonal represents identity. Extended diagonals spanning up to several kilobases indicate extensive similarities due to the recent internal duplications of this DNA in the genome of primates. In the background, besides a few random points, short imperfect diagonals in the 200-300 base pair (bp) range, form like interrupted horizontal and vertical lines and represent dispersed repeats such as Alu sequences. A model for the evolution of this gene cluster was suggested from the analysis of the distribution of similarity (14). This dot plot
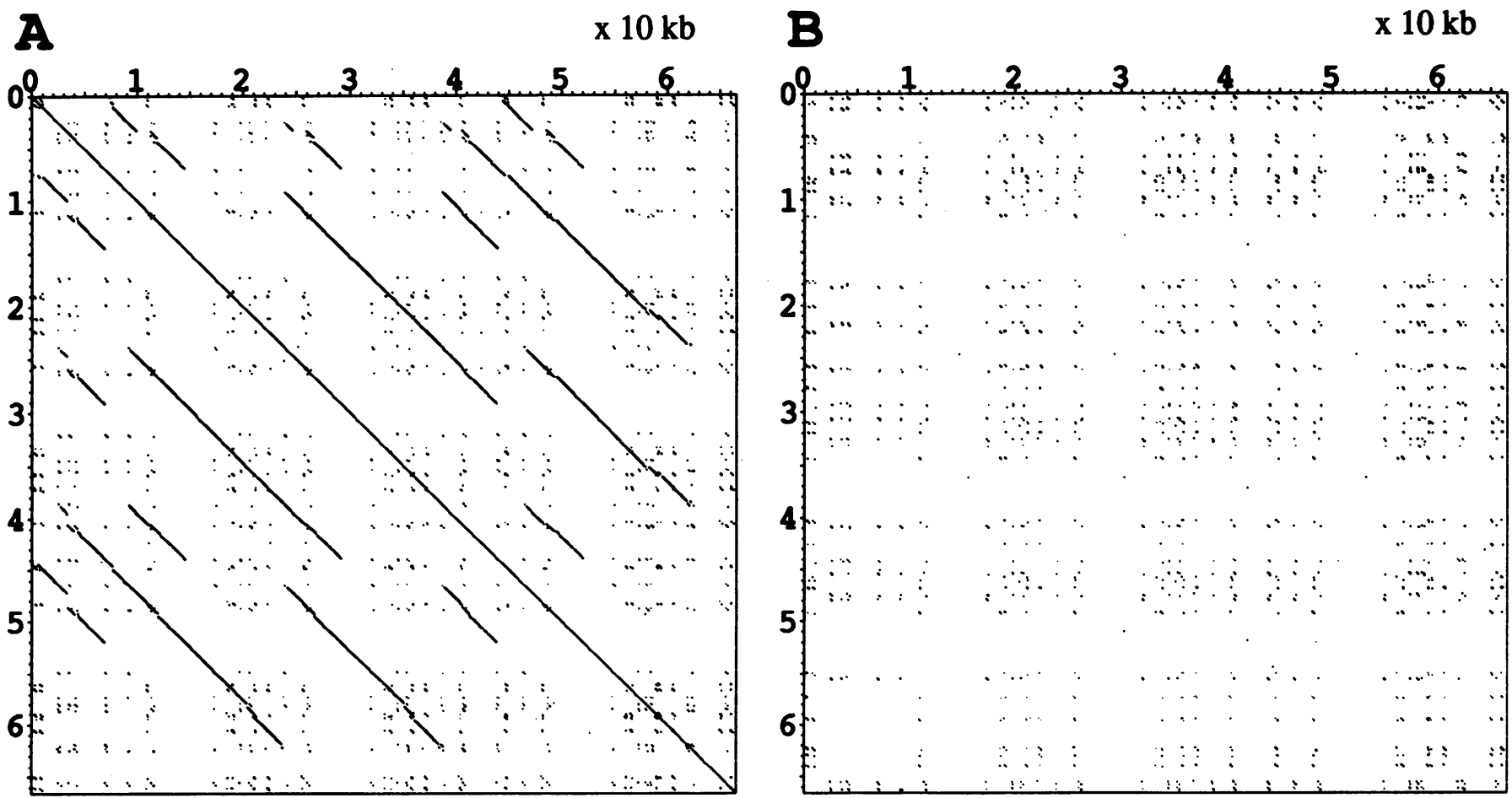

Figure 3. Similarity in the human growth hormone gene cluster (EMBL database accession no. J03071). A dot represents a perfect match of 15 nucleotides. The size of the graphic matrix is $1000 \times 1000$ dots. In $\mathbf{A}$ the sequence is compared to itself. Due to the recent multiple duplication and gene conversion events, extended diagonals are seen over regions of several kilobases. In $\mathbf{B}$ the sequence is compared to its reverse complement. Highly repeated elements such produce short diagonals aligned on the horizontal and vertical axis. 
contains 154721 points and is computed in $52 \mathrm{~s}$. This does not include the preprocessing of the sequence into the end-set position tree. This preliminary step is done at a speed of about $30 \mathrm{~kb}$ per second (10). In Figure $3 \mathrm{~b}$ the sequence is compared with its reverse complement. The plot contains 9908 points and is obtained in $3.5 \mathrm{~s}$. Dispersed repeated sequences such as the Alu type generate short imperfect diagonals in the $200-300$ bp range. Finally, when the sequence was compared to a randomized sequence of the same composition obtained by shuffling of the original sequence, the plot was computed in $1.5 \mathrm{~s}$ and only 4 random matches were found. This illustrates how sequence similarity can be found at different levels correlating with different molecular aspects of genome organization and evolution. Computation is proportional to similarity. However with our algorithm, the computation time for this large DNA is substantially better than the performance of matrix transversal algorithms, where a matrix size of $(66 \mathrm{~kb})^{2} \sim 4.4 \times 10^{9}$ matrix elements may require hours of computation.

\section{The imperfect match problem}

Perfect matching may be sufficient for the representation of similarity in closely related sequences. However, in the general case of the comparison of more distant sequences, the detection of similarity requires the consideration of approximate matches. This is generally done through the setting of a percentage of similarity allowing for misspells or mismatches only. The tree search algorithm for the localization of a word in a text can be

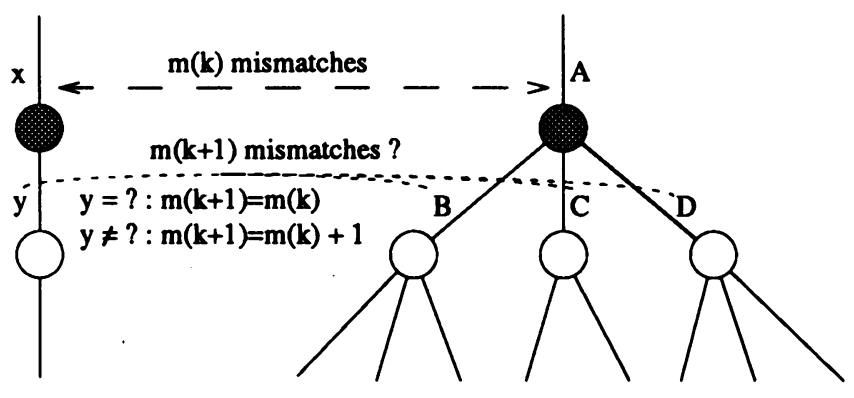

Figure 4. Pattern search algorithm. Allowing for mismatches when matching two position trees (see text for details). extended to the localization of a pattern where mismatches are permitted (12). The method is briefly illustrated in Figure 4. A word represented by node $\mathrm{N} 1$ in the position tree of S1 matches with $m$ mismatches a word in S2 represented by N2. The labels of the edges leaving $\mathrm{N} 1$ are compared successively with all the label of the edges leaving $\mathrm{N} 2$. If the labels are identical then the words in S1 and S2, represented by the nodes the edges under consideration lead to, match with $m$ mismatches. If the labels differ, then the words match with $m+1$ mismatches. In a similar manner, the procedure described above for the comparison of two positions trees can be modified to consider mismatches. Let MaxMismatch be the maximum number of mismatches permitted and suppose that a sub-word $w$ of length $k$ in sequence S1 represented by node $\mathrm{N} 1$ matches with $m$ mismatches ( $m \leq$ MaxMismatch) a sub-word in sequence $\mathbf{S} 2$ represented by node N2. (1) If $k=T$ then $w$ is a match between the two sequences and the position of occurrence can be obtained as described above from the sub-trees rooted at N1 and N2. (2) If N1 (respectively N2) is a terminal node, the $T-k$ remaining symbols from the end of $w$ can be matched against the sub-tree rooted at $\mathrm{N} 2$ (respectively N1). The only difference here is that this matching is done with the algorithm for the localization of a pattern rather than a perfect word. (3) Finally, when N1 and N2 are internal nodes in the position tree, the labels of the edges extruding from $\mathrm{N} 1$ and N2 are systematically compared. If they differ then they match with CurrentMismatch $=m+1$ mismatches otherwise they match with CurrentMismatch $=m$ mismatches. Then, if CurrentMismatch $\leq$ MaxMismatch, the nodes ND1 and ND2 the edges lead to are said to match with CurrentMismatch mismatches and the process can be called upon recursively. The procedure Match2trees is easily extended for the imperfect matching problem.

$\begin{array}{ll}\begin{array}{l}\text { Match2trees (Node1, Node2, } k, m) \\ \text {.if } k=T \text { then }\end{array} & \begin{array}{l}\text {.collect positions of occurrence of word } w \text { from } \\ \text { the sub-trees rooted at Node1 and Node2. } \\ \text {.return }\end{array} \\ \text {.match (with at most MaxMismatch }-m \\ \text { mismatches) the } T-k \text { symbols following the } \\ \text { word represented by Node1 with sub-tree } \\ \text { rooted at Node2 } \\ \text {.return } \\ \text {.match (with at most MaxMismatch - } m \\ \text { mismatches) the } T-\mathrm{k} \text { symbols following the } \\ \text { word represented by Node2 with sub-tree }\end{array}$

Table 1. Summary of similarities found in the yeast chromosome III sequence.

\begin{tabular}{|c|c|c|c|c|}
\hline Sequence & Feature & Position & Repeat label & View \& comment \\
\hline Mating Type & $\begin{array}{l}\text { MAT-alpha } \\
\text { HML-alpha } \\
\text { HML-a locus }\end{array}$ & $\begin{array}{c}197500-200000 \\
11400-14000 \\
291500-293500\end{array}$ & $\begin{array}{l}\text { repeats c \& d } \\
\text { repeats c \& d } \\
\text { repeats c \& d }\end{array}$ & $5 \mathrm{E}$ right side \\
\hline Telomeres & $\begin{array}{l}5^{\prime}-\mathrm{C}_{1-3} \mathrm{~A}-3^{\prime} \text { repeat } \\
3^{\prime} \mathrm{X} \text { left telomere } \\
\text { pseudo-X 3' } \\
\text { pseudo-X 3' } \\
5^{\prime} \mathrm{X} \text { left telomere } \\
5^{\prime} \mathrm{X} \text { right telomere } \\
\text { weak 3'X } \\
\text { weak 3'X } \\
\text { right telomere }\end{array}$ & $\begin{array}{c}1-400 \\
1200-1450 \\
4100-4350 \\
290650-290900 \\
600-1200 \\
315000-314400 \\
4500-4600 \\
291050-291260 \\
314950-315350 \\
302800-302400\end{array}$ & $\begin{array}{l}\text { repeat a } \\
\text { repeat a } \\
\text { repeat a }\end{array}$ & $\begin{array}{l}\text { 5B } \\
5 B \\
5 B \\
5 E \& 5 F \\
\text { data not shown } \\
\text { inverted repeat } \\
\text { 5B } \\
\text { 5F } \\
\text { data not shown } \\
\text { inverted repeat }\end{array}$ \\
\hline $\begin{array}{l}\text { Direct } \\
\text { repeats }\end{array}$ & $\begin{array}{l}\text { FUS1 } \\
\text { ORF YCL26C } \\
\text { YCR104w }\end{array}$ & $\begin{array}{c}73400-74000 \\
74700-75300 \\
288800-289100 \\
306550-306850\end{array}$ & $\begin{array}{l}\text { repeat } u \\
\text { repeat } u \\
\text { repeat } v \\
\text { repeat } v\end{array}$ & $\begin{array}{l}5 \mathrm{D} \\
5 \mathrm{H}\end{array}$ \\
\hline
\end{tabular}


rooted at Node1

.return

.for all edges leaving Node1 .let Edgel be that edge

.let ND1 be the node this edge leads to for all edges leaving Node2 .let Edge2 be that edge let CurrentMismatch be $m$ if the labels of Edge1 and Edge2 are different

.let CurrentMismatch be $m+1$

.if CurrentMismatch $\leq$ MaxMismatch

.let ND2 be the node Edge2 leads to

.Match2trees (ND1, ND2, $k+1$, CurrentMismatch)

.return

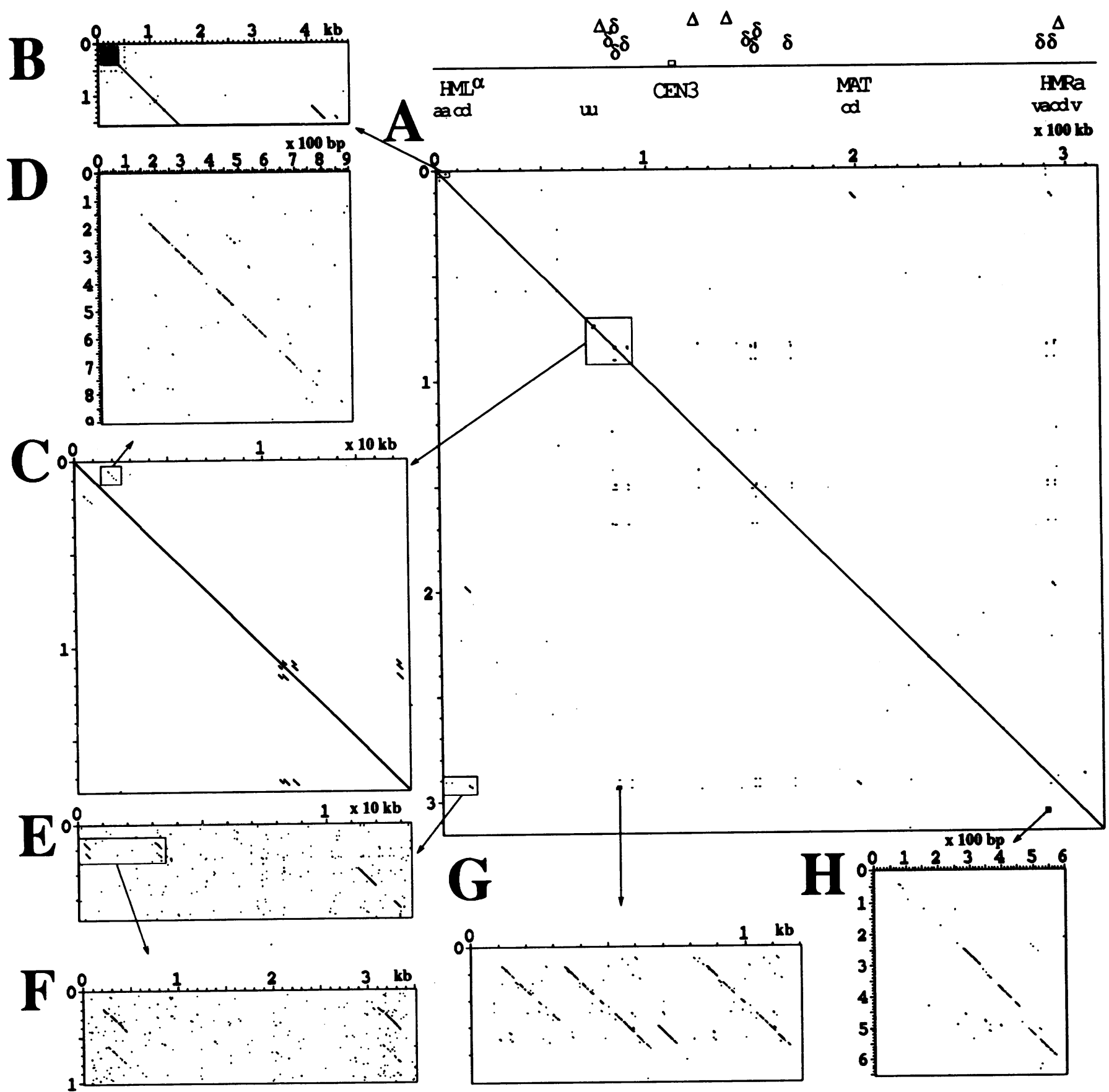

Figure 5. Similarity in the yeast chromosome III (EMBL database accession no. X59720). In A, the complete 315357 nucleotide sequence is compared to itself. A dot represent a match of 60 nucleotides containing up to 20 mismatches where the first five mismatches are not allowed to occur before a match of 5,8,11,14 and 17 nucleotides, respectively. This plot is computed in $12 \mathrm{~min}$. B-H, Recomputed sub views for specific areas of plot A. The horizontal and vertical origins are: B $(1,1), \mathbf{C}(73000,7300)$, D $(7430,73230), \mathbf{E}(900,290000), \mathbf{F}(1000,290500), \mathbf{G}(83600,293700)$ and $\mathbf{H}(288500,306300)$. The same parameters as in A are used in C. In B and D-H a dot represents a match of 42 letters containing up to 20 mismatches. For B and E, the first 6 mismatches are not permitted before a match of $3,6,9,12,15$ and 18 nucleotides, respectively. Computation takes 5 and $6 \mathrm{~s}$, respectively. D is computed in $5 \mathrm{~s}$ and the first mismatch is not allowed before a perfect match of two nucleotides. $F$ is obtained in $5 \mathrm{~s}$ with the first five mismatches not permitted before a match of $2,4,6,8$ and 10 nucleotides. For $\mathrm{G}$ and $\mathrm{H}$ the first four mismatches are not allowed before a match of $1,3,5$ and 7 nucleotides and they take 5 and $2 \mathrm{~s}$, respectively to compute. 
As the number of possible word matches for each prefix increases with the number of mismatches, execution time follows a combinatorial growth, up to a limit where all words in one sequence match all words in the other (matching with $0 \%$ similarity). In this worst case situation, the execution time varies with the product of the sequence lengths. With the intermediate situation, execution time will depend upon the number of possible prefix matches in the sequence data. One important advantage in using the position tree for word matching is that, with a refined definition of approximate similarity, execution time can be cut down drastically. It is recognized that even imperfect similarity is likely to share regions of perfect similarity (15). This consideration led Fristensky (9) to seek imperfect similarities prefixed with perfect similarity and to suggest some improvement of dot matrix programs through the use of oligomer tables. Here, this argument is developed in a more general and flexible manner. A mechanism is implemented for the filtration of patterns. It accepts user defined rules such as: 'the $N$ th mismatch may not precede the Xth symbol'. The similarity target is defined as a function of local similarity distribution over increasing prefix length. Rules are stored in a table which is used during the comparison of the edges in the procedure Match2trees. The instruction where CurrentMismatch is incremented when a mismatch is found is complemented with a test to check that the length $k$ of $w$ is compatible with the pattern definition. If it is not, simply return early from the procedure. During the tree transversal algorithm this has repercussions on the combinatorial process of accepting or rejecting a prefix match. In this case, good similarities (if they exist) can be trapped much faster by rejecting unlikely prefix.

\section{Applications: similarity in yeast chromosome III}

The largest contiguous DNA sequence available today is the complete sequence of the yeast chromosome III (16). In Figure $5 \mathrm{~A}$, the dot plot resulting from the comparison of this large sequence with itself is presented. The main diagonal represents identity and the matrix is symmetrical relatively to this diagonal. Due to the limited resolution of the graphic printer, a point on the axis represents about 400 nucleotides. The use of such dot plots for the inference of similarity in large genomic sequences is limited by the resolution of the graphic device. As DNA data of substantial length accumulates rapidly, this will always remain a problem. Perhaps the main motivation behind our approach, is to provide a highly interactive and flexible tool for the analysis of similarity at different scales. The advantage of a good pattern filtering is to reduce background signal to a minimum. Using a stringent similarity target in a large dot matrix allows the user to localize potential regions of interest, even if at the level of graphic resolution considered, interesting regions may be represented by a single point in the matrix. These regions can then be zoomed in with better resolution and/or a less stringent similarity target for a closer analysis in record time. This aspect is illustrated in Figure $5(\mathrm{~B}-\mathrm{H})$ where close views of several discrete area of the dot matrix of Figure 5A are presented. A summary of the sequence features is presented in Table 1.

The main region of similarity can be clearly identified extending over $2 \mathrm{~kb}$. It represents the identity between the HMR-alpha and MAT-alpha loci involved in the switching mechanism of the mating type (17). This similarity identifies the 'alpha' type for this chromosome. Another imperfect similarity involves these two loci with the HML-a locus which is the other component of the mating type domain conferring the 'a' type when occupying the MAT locus.

The first $400 \mathrm{bp}$ of the left extremity of chromosome III is composed of the telomeric repeat unit $5^{\prime}-\mathrm{C}_{1-3} \mathrm{~A}-3^{\prime}$ producing a dark square on the plot. Three regions of similarity with the $\mathrm{X}$ class telomeric sequence as well as two shorter and weaker similarities were found on this chromosome. Previous reports describe the strong similarities with the first three regions, but failed to identify the lower similarities $(16,18)$. Interestingly, the recurrence of similarity with the $\mathrm{X}$ sequence, present in all telomeres of yeast, led Oliver and co-authors (16) to propose that chromosome III has grown during evolution by recombination events involving its telomeres. It is noteworthy in this context that the weaker similarities are directly adjacent to the pseudo-X regions. This apparent tandem repeat organization of internal X class sequence may be relevant to the mechanism of chromosome evolution. To search for inverted repeats, the sequence was compared to its reverse complement (data not shown). X sequence similarity was found between the left and right extremities. The last 400 nucleotides of the chromosome III sequence also show similarity with an internal region.

Two internal imperfect direct repeats were found in chromosome III (Figure 5D and 5H). A previous study failed to identify these repeats (18). These similarities probably originated through duplications.

\section{Delta elements}

The delta sequence is a 330 bp DNA sequence occurring about at least 100 times in the yeast genome (19). They are found either as isolated solo delta elements or as the terminal repeats flanking the yeast transposon (Ty) elements. Solo delta sequences result from the excision of Ty elements after delta-delta recombination and are believed to decay slowly in the genome through the accumulation of mutations. Old transposon remnants may provide a fertile substrate for the evolution of new DNA sequences and this contribution of transposons to genome evolution has been described as the 'Ty cycle' (20). In the yeast chromosome III, 14 delta elements are present: 10 on the forward strand and 4 on the reverse strand. These 14 elements can all be identified from the dot matrix presented in Figure 5A. In Figure 5C and $5 \mathrm{G}$, an enlargement of the plot shows that one of these delta elements was inserted inside another (20).

A search for weaker similarity was conducted in order to identify ancient delta elements and evaluate their contribution to the 'Ty cycle'. The 14 delta sequences were concatenated and used to analyze similarity with the yeast chromosome III. With this 'consensus' approach, a match can be considered more significant if it involves several delta sequences, producing vertically aligned dots on the plot. Confidence can be improved with this method when looking at weak similarities. In the direct orientation, a $50 \%$ similarity region of 120 nucleotides is found at position 294660 (Figure 6a). It matches 4 of the delta sequences and is directly adjacent to a reverse delta at 294800 . Two extra regions of short similarity, 150 and 110 nucleotides in length, matching with 6 and 8 of the inverted delta sequences were revealed at position 226300 and 291350 , respectively (Figure $6 c$ ). These sequences are the best candidates for remnant delta sequences. A weak candidate is also suggested from Figure 6 a around position 204900.

The analysis of similarity in the yeast chromosome III sequence illustrates the performance of the algorithm for the analysis of 


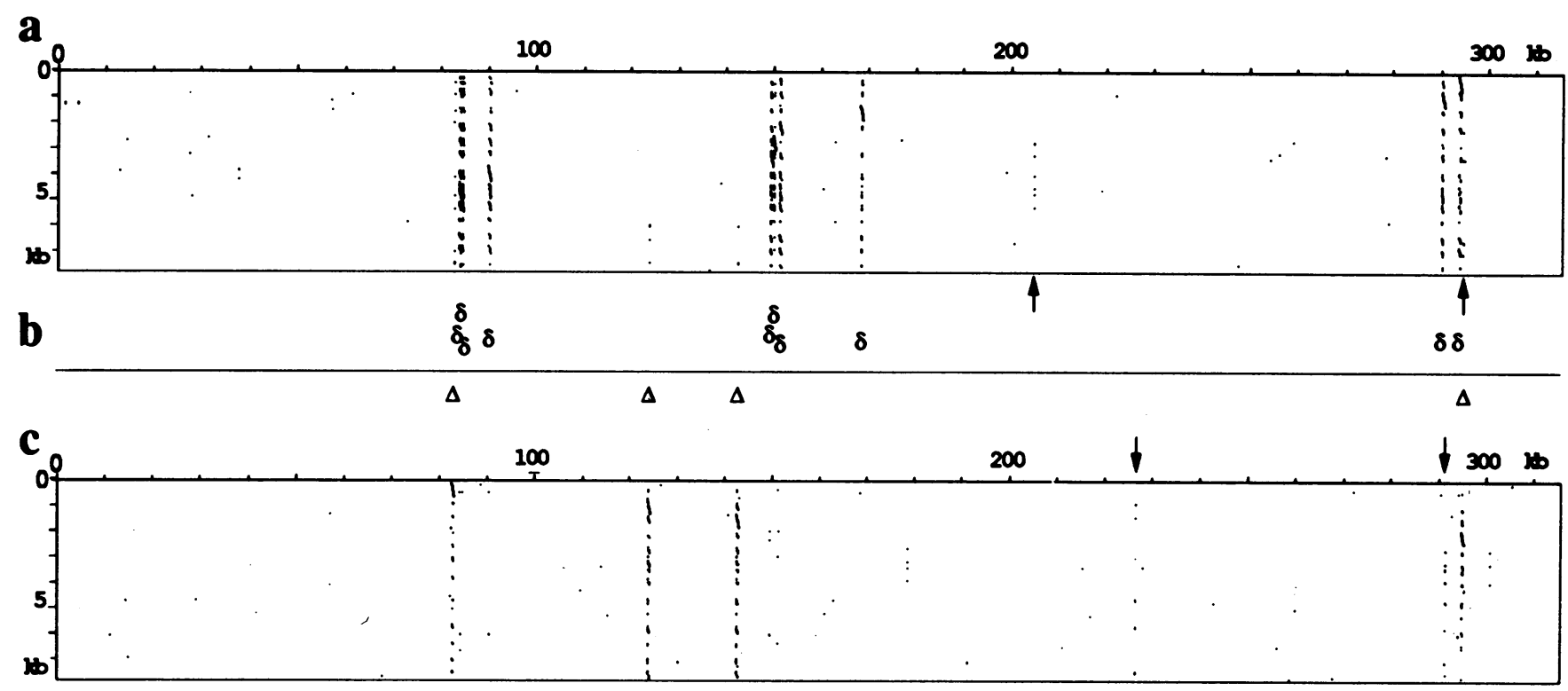

Figure 6. Consensus delta element similarities in yeast chromosome III. The complete yeast chromosome sequence (top) is compared to a $7.8 \mathrm{~kb}$ concatenate made of $600 \mathrm{bp}$ fragments from the same sequence containing a delta element inferred from the comparison in Figure 5A. The fragments were rearranged to contain the delta sequence in a consensus orientation. The central positions in the original sequence are: $293900,299400,168500,151400,150100,149500,89900,84500$, 83900 for the delta sequence found in the direct orientation and 294850, 142700, 123900, 82700 for those found in the reverse orientation. (a) Comparison in the direct orientation. (c) Comparison in the reverse orientation. A dot represent a match of 100 nucleotides containing up to $50 \%$ similarity when the $n$th mismatch is not permitted before $2 n+1$ nucleotides. Computation time is about $15 \mathrm{~min}$. The arrows indicate regions of short imperfect similarity with 6 and 8 delta sequences representing potential delta remnants. (b) Map of delta elements (d: direct delta, D: reverse delta, arrow: potential delta remnant).

large genomic DNA in connection with the biological interests in genome organization and evolution. With the use of adapted parameters for the sequence under consideration, significant similarities can be identified in acceptable computational time.

\section{DISCUSSION}

Due to the mechanisms of transmission and evolution of molecular sequences, the analysis of similarities is a fundamental method to investigate the biological significance of genetic sequences. As DNA sequences are uncovered at an exponential rate and larger sequences are assembled under diverse genome sequencing initiatives, new problems are encountered in the manipulation and analysis of this data. This paper is mainly concerned with the problem arising from the complexity of comparing large sequences where previous algorithms were plagued by a quadratic increase in computation time. Other authors have also considered this problem. They described methods based on dynamic programming for the representation (21) and the calculation of a robustness measure of similarities (22). This paper explores a different approach. It describes a word search algorithm for the representation of similarities between two sequences and proposes the implementation of simple 'user defined' heuristics for efficient pattern matching leading to a better control of computational complexity. The algorithm uses the position tree as an index to the word composition of the sequence data. The position tree occupies space that grows only linearly with sequence length, but it needs to be kept in central memory. With our implementation, one megabyte of memory is necessary for every $27 \mathrm{~kb}$ of DNA sequence. Until recently, the lack of large random access memories made this approach unrealistic. However, in the last few years, technological progress has made these devices available on the microcomputer and even further improvements are expected soon. This coincidence between DNA sequencing and improved computer memory technology provides a stimulating environment for the further development of the use of automata in genetic sequence analysis.

The algorithm for perfect matching is very fast as it incorporates optimal recursive procedures for the localization of word occurrence (13). The time penalty for imperfect matching is heavy. This can be partly attributed to the noise inherent in the process of pattern matching, simply because sequences tend to be more alike when errors are permitted. However, the implementation of adequate decision rules about the prefixing of similarity provides a rationale for the filtration of this noise and the control of computation complexity. Here, because the program is designed to be highly interactive, the choice of decision rules is left to the user's decision. Acceptable rules may be determined empirically, depending upon the time the user wants to invest, the resolution expected and the size of the problem considered. From the authors experience, specific rules influence drastically computation time with a relatively minor impact on the detection of similarity. This is particularly true for long sequences where, due to the limits of graphic resolution, the extension of a region of similarity by more relaxed parameters are imperceptible. However, a better theoretical framework for the selection of rules should be developed. The effects of the alphabet size, the sequence length, the tuple parameter and the number or errors should be quantified. Optimal sets of rules could then be deduced for different similarity targets. With such a model, the program could be modified to become entirely automatic. It would provide a fast representation of the most significant similarity in a first optimal pass, then more time can be spent exploring decreasing similarities in successive passes. 
The performance of the program has been illustrated with the analysis of similarity in large genomic DNA. Previously unreported repeats, delta element and delta remnants have been identified in the yeast chromosome III. As the genome sequencing initiative matures into the archiving of life history in the form of DNA sequences, the program 'Fast-Dots' might provide an interactive tool for the exploration of genome organization and evolution on the microcomputer. To obtain a copy of 'Fast-Dots' send a macintosh formatted disk with your request.

\section{ACKNOWLEDGEMENTS}

The authors thank Dr Kim O'Hoy for helpful comments on the manuscript and the referee for making useful suggestions that improved the presentation of this paper substantially.

\section{REFERENCES}

1. Fitch, W.M. (1969) Biochem. Genet. 3, 99-108.

2. Gibbs, A.J. and McIntyre, G.A. (1970) Eur. J. Biochem. 16, 1-11.

3. McLachlan, A.D. (1971) J. Mol. Biol. 61, 409-424.

4. Maizel, J.V., Jr and Lenk R. P. (1981) Proc. Natl. Acad. Sci. USA 78, 7665-7669.

5. Steinmetz, M., Frelinger, J.G., Fischer, D., Hunkapillar, T., Periera, D., Weissman, S.M., Uehara, H., Nathenson, S. and Hood, L. (1981) Cell 24, $125-134$.

6. Novotny, J. (1982) Nucleic Acids Res. 10, 127-131.

7. Harr, R., Hagblom, P. and Gustafsson, P. (1982) Nucleic Acids Res. 10, $365-374$.

8. Staden, R. (1982) Nucleic Acids Res. 10, 2951-2961.

9. Fristensky, B. (1986) Nucleic Acids Res. 14, 597-610.

10. Lefevre, C. and Ikeda, J.E. (1993) CABIOS 9, 343-348.

11. Aho, A.V., Hopcroft, J.E. and Ullman, J.D. (1974) The Design and Analysis of Computer Algorithm. Addison-Wesley, Reading, MA.

12. Lefevre, C. and Ikeda, J.E. (1993) CABIOS (1993b) 9, 349-354.

13. Blumer, A., Blumer, J., Haussler, D. and McConnell, R. (1987) J. ACM 34, 578-595.

14. Chen E.Y., Liao Y.C., Smith D.H., Barrera-Saldana H.A., Gelinas R.E. and Seeburg P.H (1989) Genomics 4, 479-497.

15. Wilbur, W.J. and Lipman, D.J. (1983) Proc. Natl. Acad. Sci. USA 80, 726-730.

16. Oliver, S.G. et al. (1992) Nature 357, 38-46.

17. Herskowitz, I. (1989) Nature 342, 749-757.

18. Karlin, S., Blaisdell, B.E., Sapolsky, R.J., Cardon, L. and Burge, C. (1993) Nucleic Acids Res. 21, 703-711.

19. Roeder, G.S. and Fink, R.F. (1983) Transposable elements in yeast. In Mobile Genetic Elements. Academic Press Inc.

20. Fink, G. R., Boeke, J.D. and Garfinkel, D.J. (1986) Trends Genet. 2, $118-123$.

21. Schwartz, S., Miller, W., Yang, C.M. and Hardison, R.C.(1991) Nucleic Acids Res. 19, 4663-4667.

22. Chao, K.M., Hardison, R.C. and Miller, W. (1993) CABIOS 9, 387-396. 